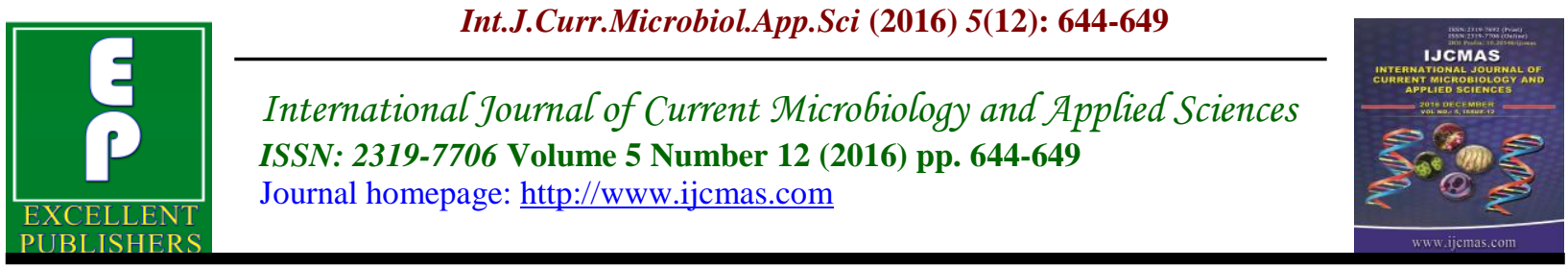

Original Research Article

http://dx.doi.org/10.20546/ijcmas.2016.512.071

\title{
Prevalence and Recent Trend in the Antibiogram of Staphylococcus Species Isolated from Clinical Specimens in a Rural Tertiary Care Hospital in South India
}

\author{
Ruby Thomas*, K. Shilpa and Allavarapu Ramyashree \\ Department of Microbiology, Akash Institute of Medical Sciences and Research Center, \\ Prasannahalli road, Devanahalli, Bengaluru rural-562110, India \\ *Corresponding author
}

Keywords

Staphylococcus

species,

antibiotic

susceptibility,

prevalence,

resistance, MRSA.

Article Info

Accepted:

20 November 2016

Available Online:

10 December 2016

\section{A B S T R A C T}

The present study was carried out to determine the prevalence of Staphylococcus species in clinical samples and to find out the trend of susceptibility to antibiotics in a rural setting. Standard microbiological and biochemical methods were used to screen 503 clinical specimens comprising of sputum, pus, urine and blood for Staphylococcus species. One thirty nine isolates were obtained from these samples. Of the 64 positive isolates from pus, 19 were Methicillin sensitive Coagulase negative Staphylococcus species (MS-CoNS), 17 Methicillin resistant Coagulase negative Staphylococcus species (MR-CoNS), 24 Methicillin sensitive Staphylococcus aureus (MSSA) and 4 Methicillin resistant Staphylococcus aureus (MRSA). Out of the 30 isolates from urine 12 MS-CoNS, 10 MR-CoNS, 6 MSSA and 2 were MRSA. Blood grew 17 MS-CoNS, 12 MR-CoNS ,6 MSSA and no MRSA. The 10 sputum positive samples had 9 MSSA and 1 MRSA. Antibiotic susceptibility testing was done for all the positive isolates. Linezolid has been found to be the most sensitive to all the four categories followed by Gentamicin. For MSSA, Clindamycin and Erythromycin has been found to be equally sensitive (53\%). Penicillin is the least effective drug for all the 4 categories. All the MRSA were sensitive to Vancomycin. Also it was noted that there was a reduction in the prevalence of MRSA (5.03\%) from this area.

\section{Introduction}

Staphylococcus is a common bacterium found in clinical samples (Diekema et al., 2001) and also found to cause human infections (Lowy, 1998). Staphylococcus aureus ( $S$. aureus) being the most important member of this group (Javid et al., 2006) usually produces skin infections and if left untreated it may progress to a wide range of complications like wounds, tissue infections, pneumonia, joint and /or bone infections (Delorme et al., 2009). It is also the most frequently encountered species in the hospitals (Emmerson et al., 2004). The hospital acquired infections includes wound infections, ventilator associated pneumonia, blood stream infections caused by intravenous devices and prosthetic materials. The major reservoir of Staphylococcus 
aureus in hospitals are colonised and infected patients. Health care workers and patients are the main source of Staphylococcus aureus infections. Methicillin was introduced in 1961 and was the first semi synthetic penicillinase resistant drug. S.aureus after that showed a wide spread resistance to Penicillin (Jevons et al., 1961). This further lead to the emergence of Methicillin resistance spreading globally across hospitals. This Methicillin resistance to S.aureus is spreading not only in hospitals but also in community settings. Methicillin resistance is also seen in several other species like S.epidermidis, S.haemolyticus, S.hominis and S.capitis. (Peacock et al., 2005).

\section{Materials and Methods}

This study was carried out at Akash hospital, Bangalore Rural North between January 2015 to October 2016 which serves as a tertiary care hospital in a rural area. From a total of 503 samples processed from pus, urine, blood and sputum, we have obtained 139 clinical isolates of all species of Staphylococcus. S.aureus (both Methicillin resistant and Methicillin sensitive) and CoNS (both Methicillin resistant and sensitive) were obtained from the above mentioned samples. These clinical samples were inoculated on Blood agar and Mac Conkey agar incubated at $37^{\circ} \mathrm{C}$ aerobically for 24 hours.

The isolates were subjected to antibiotic testing by the Kirby Bauer disc diffusion method on Mueller Hinton agar plates as per Clinical Laboratory Standards Institute guidelines (CLSI) 2014. The antibiotics used were Cefoxitin(30 $\mu$ g), Penicillin(10units), Erythromycin $(15 \mu \mathrm{g})$, Clindamycin $(2 \mu \mathrm{g})$, Gentamicin $(10 \mu \mathrm{g}), \quad$ Linezolid $\quad(30 \mu \mathrm{g})$, Cotrimoxazole $(25 \mu \mathrm{g})$, Ciprofloxacin $(30 \mu \mathrm{g})$ and Vancomycin $(30 \mu \mathrm{g})$ for MRSA. MRSA was determined showing resistance to Cefoxitin disc while inducible Clindamycin resistance was detected by perfoming the D test following CLSI 2014.

\section{Results and Discussion}

The distribution pattern of Staphylococcal species from all clinical samples is shown in Table 1. Methicillin resistance to. S.aureus was seen in $5.03 \%$ of the isolates (5 from pus, 1 from urine and 1 from sputum) all were Vancomycin sensitive. MR-CoNS made up $28.05 \%$ of the isolates (17 from pus, 10 from urine, and 12 from blood samples), out of which some may be contaminants which were also Vancomycin sensitive. Vancomycin tested by the disc diffusion method however can produce erratic results. D test was positive in four MSSA isolates that indicates inducible resistance to Clindamycin (ICR).

Of the 139 isolates there was female preponderance in age group between, 16 to 30 yrs as shown in Table 3.

Drug resistance pattern to several antibiotics was different among the isolates as shown in Figures 1, 2, $3 \& 4$. There was also a significant reduction in the number of MRSA isolates $(5.03 \%)$ as shown in Table 1.

In case of MRSA as shown in Figure 4, out of the 9 antibiotics used Linezolid and Vancomycin works the best with $100 \%$ sensitivity. Vancomycin is the drug of choice for MRSA.

Staphylococcus is ubiquitous and has a remarkable adaptability, and versatility to exist as a commensal and pathogen. It also is one of the most infectious agent with high prevalence in both hospital and community settings (Akindele et al., 2010). 
Table.1 Staphylococcus Species Isolated From Various Clinical Samples

\begin{tabular}{|l|c|c|c|c|}
\hline Specimens & MS-CoNS & MR-CoNS & MSSA & MRSA \\
\hline Urine & $12(25 \%)$ & $10(25.64 \%)$ & $6(13.33 \%)$ & $2(28.57 \%)$ \\
\hline Pus & $19(39.58 \%)$ & $17(43.58 \%)$ & $24(53.33 \%)$ & $4(57.14 \%)$ \\
\hline Blood & $17(48.57 \%)$ & $12(34.28 \%)$ & $6(17.14 \%)$ & 0 \\
\hline Sputum & 0 & 0 & $9(90 \%)$ & $1(10 \%)$ \\
\hline Total & $48(34.53 \%)$ & $39(28.05 \%)$ & $45(32.37 \%)$ & $7(5.03 \%)$ \\
\hline
\end{tabular}

Table.2 Age Group of Patients Infected With MS-CoNS, MR-CoNS, MSSA, MRSA

\begin{tabular}{|l|l|l|l|l|l|}
\hline Charasteristics & $\begin{array}{l}\text { Total } \\
\text { cases (\%) } \\
(\mathbf{n = 1 3 9})\end{array}$ & $\begin{array}{l}\text { MS-CONS } \\
\text { Cases (\%) } \\
(\mathbf{n = 4 8})\end{array}$ & $\begin{array}{l}\text { MR-CONS } \\
\text { Cases (\%) } \\
(\mathbf{n = 3 9})\end{array}$ & $\begin{array}{l}\text { MSSA } \\
\text { Cases (\%) } \\
(\mathbf{n = 4 5 )}\end{array}$ & $\begin{array}{l}\text { MRSA } \\
\text { Cases (\%) } \\
(\mathbf{n = 7})\end{array}$ \\
\hline Male & $60(43.16)$ & $18(37.5)$ & $15(38.46)$ & $20(44.44)$ & $3(42.85)$ \\
\hline $\begin{array}{l}\text { Female } \\
\text { Paediatrics } \quad(<\mathbf{1 8} \\
\text { years old) }\end{array}$ & $37(56.83)$ & $30(62.5)$ & $24(61.53)$ & $25(55.55)$ & $4(57.14)$ \\
\hline $\begin{array}{l}\text { Adults }(>=18 \text { years } \\
\text { old) }\end{array}$ & $102(73.38)$ & $30(62.5)$ & $27(69.23)$ & $30(66.66)$ & $7(100)$ \\
\hline
\end{tabular}

Table.3 Frequency of Age and Sex Wise Distribution

\begin{tabular}{|l|l|l|}
\hline Age in years & $\begin{array}{l}\text { Males } \\
(\mathbf{N = 6 0})\end{array}$ & $\begin{array}{l}\text { Females } \\
(\mathbf{N = 7 9})\end{array}$ \\
\hline $\mathbf{0 - 1 5}$ & 15 & 16 \\
\hline $\mathbf{1 6 - 3 0}$ & 10 & 29 \\
\hline $\mathbf{3 1 - 4 5}$ & 14 & 13 \\
\hline $\mathbf{4 5 - 6 0}$ & 14 & 14 \\
\hline $\mathbf{8 0}$ & 07 & 07 \\
\hline
\end{tabular}

Fig.1 Methicillin sensitive Coagulase negative Staphylococcus (MS-CoNS)

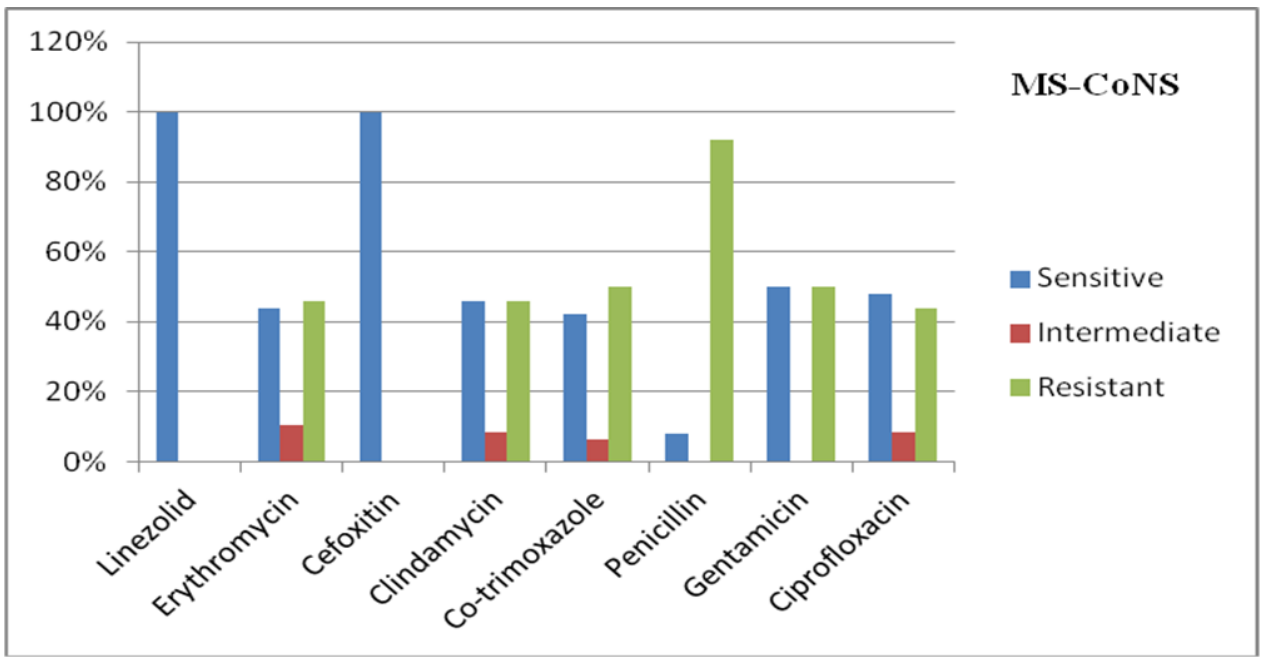


Fig.2 Methicillin resistant Coagulase negative Staphylococcus (MR-CoNS)

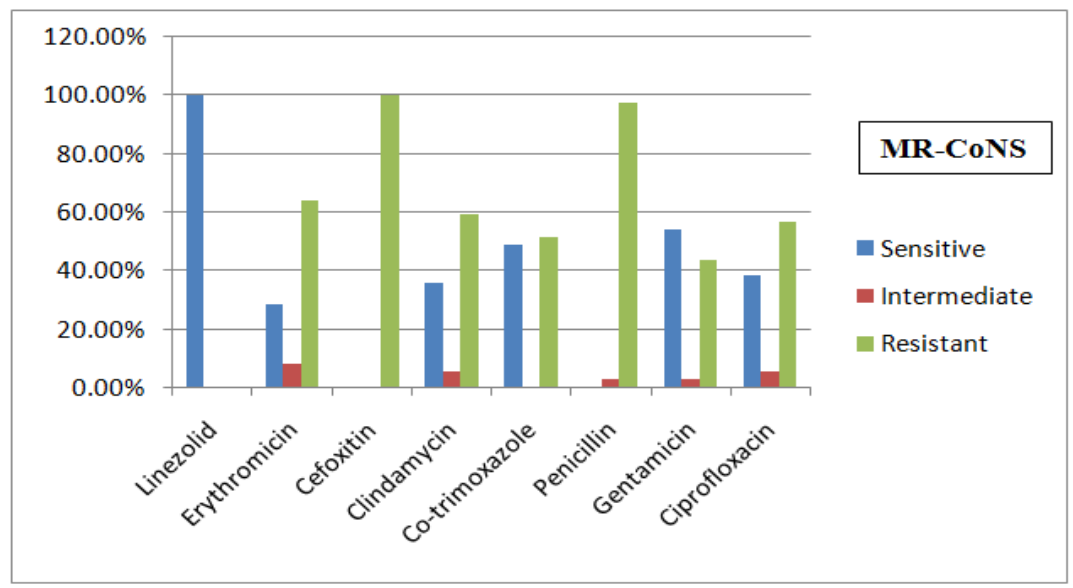

Fig.3 Methicillin sensitive Staphylococcus aureus (MSSA)

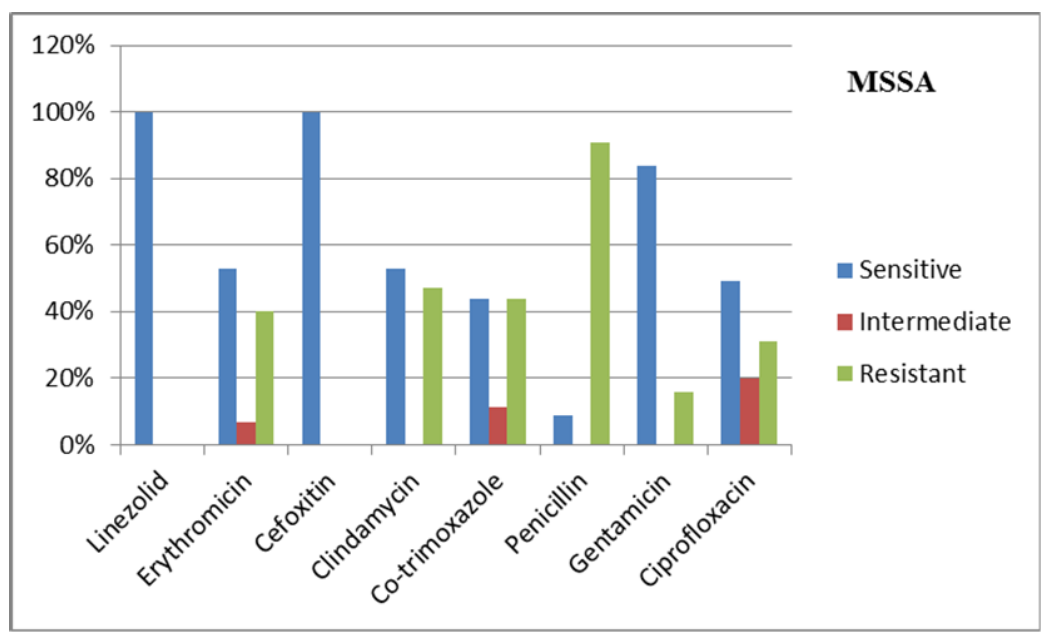

Figure.4 Methicillin resistant Staphylococcus aureus (MRSA)

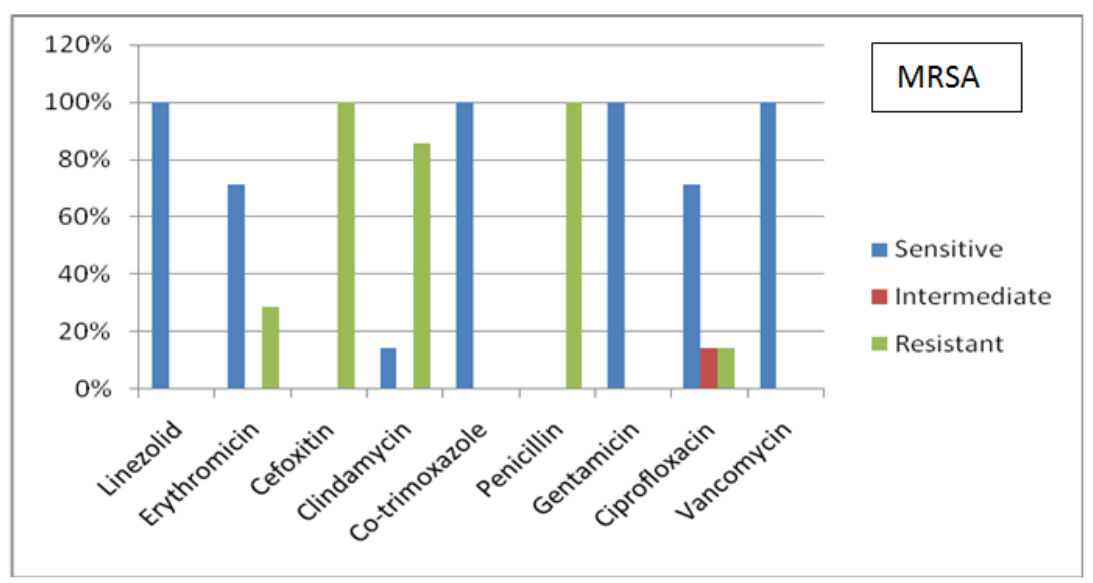


This study reports the isolation and characterisation of S.aureus (both Methicillin sensitive and resistant) and CoNS (Methicillin sensitive and resistant) from clinical samples at the tertiary care hospital. A total of 139 isolates which were from pus, urine, blood and sputum were processed. The highest number of isolates of S.aureus was from pus which was 28 out of $139(20.14 \%)$ followed by 10 from sputum (7.19\%), 8 from urine $(5.75 \%)$ and 6 from blood $(4.31 \%)$. In our study there was an increase in isolation of MS-CoNS which was 48 out of $139(34.53 \%)$ in comparison with 45 of MSSA (32.37\%), findings were consistent with reports elsewhere (Orji et al., 2012). Some of the CoNS could have been contaminants or opportunistic pathogens (Nworie and Umeh, 2010). This could be due to poor personal hygiene of the patients from this locality who treat themselves or go elsewhere to quacks before seeking medical attention which could lead to colonisation of the wounds.

Methicillin sensitive Staphylococcus aureus (MSSA) was found to be $100 \%$ sensitive to Linezolid, 84\% sensitive to Gentamicin ,and 53\% sensitive to both Erythromycin and Clindamycin, $49 \%$ sensitive to Ciprofloxacin, $44 \%$ sensitive to Cotrimoxazole and least susceptible to Penicillin (9\%). Methicillin sensitive Coagulase negative Staphylococcus (MSCoNS) was also found to be most sensitive to Linezolid (100\%) followed by Gentamicin (50\%) Ciprofloxacin (48\%) Clindamycin (46\%), Erythromycin (44\%), Cotrimoxazole (42\%), least susceptible to Penicillin (8\%), and MRSA was found to be $100 \%$ sensitive to Vancomycin and Linezolid.

The present study concluded that $53.53 \%$ of MSSA from pus (from wounds and ear swab) which showed excellent susceptibility to Linezolid (100\%) followed by Gentamicin (84\%) Ciprofloxacin (49\%), Cotrimoxazole (44\%) and least susceptibility to Penicillin (9\%), Clindamycin sensitivity was found to be effective only for MSSA (53\%). MRSA shows $100 \%$ sensitivity to Vancomycin. There was a reduction in MRSA (5.03\%) as compared to other studies probably as this is a rural area where patients are less exposed to antibiotics compared to urban areas. Considering the limited number of antibiotics to which different species of Staphylococcus were sensitive it is mandatory to follow good infection control practices and also adopt an effective antimicrobial stewardship programme for the hospital.

\section{References}

Akindele, A.A., Adewuyi, I.K., Adefioy, O.A., Adedokun, S.A., Olaolu, A.O. 2010. Antibiogram and BetaLactamase production of Staphylococcus aureus Isolates from Different Human Clinical Specimens in a Tertiary Health Institution in lleife, Nigeria. American-Eurasian J. Sci. Res., 5(4):230-233, 2010.

Clinical and Laboratory Standard Institute, "Performance standards for antimicrobial disk susceptibility tests", Approved standard, Document M100S23, Clinical and Laboratory Standards Institute, Wayne, pa, USA, 2014.

Delorme, T., Rose, S., Senita, J., Callahan, C., and Nasr, P. 2009. "Epidemiology and susceptibilities of methicillinresistant Staphylococcus aureus in Northeastern Ohio," The American J. Clin. Pathol., vol. 132, no. 5, pp. 616687.

Diekema, D.J., Pfller, M.A., Schmitz, F.J., Smayevsky, J., Bell, J., Jones, R.N., 
Beach, M. 2001. The Sentry participants Group: Survey of infections due to Staphylococcus species: frequency of occurance and antimicrobial susceptibility of isolates collected in United States, Canada, Latin America, Europe and Western Pacific Region for the SENTRY Antimicrobial Surveillance program, 1997-1999. Clin. Infect. Dis., 32: 114132.

Emmerson, M. 2004. Nosocomial Staphylococcal outbreak. Scandinavian J. Infect. Dis., Suppl; 93: 47-54.

Jevons, M.P. 1961. Celbenin-resistant Staphylococci. Br. Med. J., 1: 124-125. Javid, A.D., Manzoor, A.T., Jamal, A.K., Asif, A., Mohammed, A.K., Mohammed, K., Khalid, H.B., Mohammad, J.D., Niyaz, A., Shamim. A. 2006. Molecular epidemiology of clinical and carrier strains of methicillin resistant Staphylococcus aureus (MRSA) in the hospital settings of north India. Annals of Clin. Microbiol. Antimicrobials, 5: 22.

Lowy, F.D. 1998. Staphylococcus aureus infection. N. Engl. J. Med., 339:520532.

Nworie, A., Umeh, N. 2010. Investigation of Urinary Tract Infection among High School Children in Abakaliki Metropolis. J. Biomed. Sci. Nig., Vol. 8 No. $1 \& 2$.

Orji, L., Nworie, A., Eza, U.A., Agberotimi, 1.O., Okereke, E.C., Azi, S.O. 2012. The prevalence and antimicrobial susceptibility profile of methicillinresistant Staphylococcus aureus isolates from clinical specimens in a Tertiary Hospital, South East Nigeria. Continental J. Pharmaceutical Sci., 6(1): 23-29.

Peacock, S.J. 2005. Staphylococcus. In: Murray PR, Bureau S, Koster J, Vandenberg L, Pengilley Z, editors. Topley \& Wilson's Microbiology \& Microbial infections. 10th ed. London: Hodder Arnold, p. 769-70.

\section{How to cite this article:}

Ruby Thomas, K. Shilpa and Allavarapu Ramyashree. 2016. Prevalence and Recent Trend in the Antibiogram of Staphylococcus Species Isolated from Clinical Specimens in a Rural Tertiary Care Hospital in South India. Int.J.Curr.Microbiol.App.Sci. 5(12): 644-649. doi: http://dx.doi.org/10.20546/ijcmas.2016.512.071 